\title{
Review
}

\section{F-18 FDG PET/PET-CT in the Management of Lymphoma}

\author{
HALANAIK DHANAPATHI AND RAKESH KUMAR
}

\begin{abstract}
Positron emission tomography (PET) provides metabolic information and is superior to CT scan. The major utilization of PET is in oncology. Most commonly used PET tracer is fluorine 18 fluorodeoxyglucose (FDG), an analogue of glucose. This isotope accumulates in most tumours in a greater amount than it does in normal tissue. Malignant lymphomas are a heterogeneous group of diseases whose treatment and prognosis depend on accurate subtype and staging. F-FDG PET scan has been found useful in the staging and follow-up of Hodgkin's and non-Hodgkin's lymphoma. Here we review role of FDG PET in management of lymphoma.
\end{abstract}

INTRODUCTION:

Lymphoma is a common hematopoetic malignancy. Accurate diagnosis, correct staging and proper therapy is important for successful outcome.

Ann Arbor's staging is commonly used for both Hodgkin's and non Hodgkin's Lymphoma. Patients are staged from I to IV and each stage number is divided into A (absence) or B based on presence of symptoms such as - unexplained fever above $38^{\circ} \mathrm{C}$, night sweats and weight loss more than $10 \%$ body weight within preceding 6 months. For staging - apart from thorough clinical examination, radiological investigations-chest X-Ray and CT Scan of chest and abdomen are employed. ${ }^{1}$

Positron emission tomography (PET) is a functional imaging modality. ${ }^{18} \mathrm{~F}$ - fluoro-2-deoxyD-glucose (FDG) is the most commonly used radiotracer in PET imaging. FDG is an analogue

Department of Nuclear Medicine, AIIMS, New Delhi-110029

Correspondence to: RAKESH KUMAR

E-mail: rkphulia@hotmail.com of glucose and the uptake is directly proportional to the glucose metabolism of tumour tissue. Malignant tumours with high glucose metabolism show preferential uptake of FDG as compared to surrounding normal cells. After transport into tumor cell, FDG is phosphorylated by hexokinase into FDG-6phosphate, which cannot continue through glycolysis because it is not a substrate for enzyme glucose-6-phosphate isomerase. As a result, ${ }^{18} \mathrm{~F}$-FDG-6-phosphate is biochemically trapped within the cell. ${ }^{18} \mathrm{~F}$-FDG-PET is now an established standard in the initial staging, monitoring the response to the therapy, and restaging after treatment of patients with Hodgkin's lymphoma (HL) and high-grade nonHodgkin's lymphoma (NHL).

The widespread use of PET/CT takes advantage of the excellent anatomic resolution of CT Scan and the biologic characterization provided by FDG-PET which together results in improvement in accuracy of detecting the extent of disease, response evaluation and prognostication. ${ }^{1}$ PET-CT also has increased sensitivity and specificity. Its usefulness for the staging of slow-growing lymphomas has not been established.

\section{INITIAL DIAGNOSIS}

FDG-PET Scan to complement CT Scan can be used for initial staging. PET is more sensitive than specific for the diagnosis of lymphoma. False-negative scans can be obtained in cases of malignant lymphoma that are not highly metabolically active. Lymphomas arising from mucosal-associated lymphoid tissue (i.e., MALT lymphomas) are an established cause of falsenegative PET scans, and images in patients with these tumours must be interpreted with caution. ${ }^{3}$ False-positive PET scans pose a frequent hazard in interpretation and are commonly seen in patients with sarcoidosis, 
tuberculosis, histoplasmosis and other fungal infections, pyogenic abscess, and spondylodiscitis.

\section{INITIAL STAGING}

Staging of lymphoma is based on history, physical examination, laboratory tests, imaging with CT Scan, MR imaging or other conventional imaging modalities, bone marrow biopsy etc. One of the major limitations of CT scanning in lymphoma is that recognition of nodal involvement depends almost entirely on determining the size of the lymph node. Therefore, the accuracy of anatomic imaging in the identification of disease in normal-sized lymph nodes or in the detection of nonmalignant disease within enlarged nodes is limited. ${ }^{7}$ Role of ${ }^{18} \mathrm{~F}$-FDG-PET in lymphoma imaging has been well established. Detection of more extensive disease by PET than by conventional imaging would be of major relevance for patients with apparently limited stage HL in whom upstaging could alter management. (Fig. 1-2). It also may
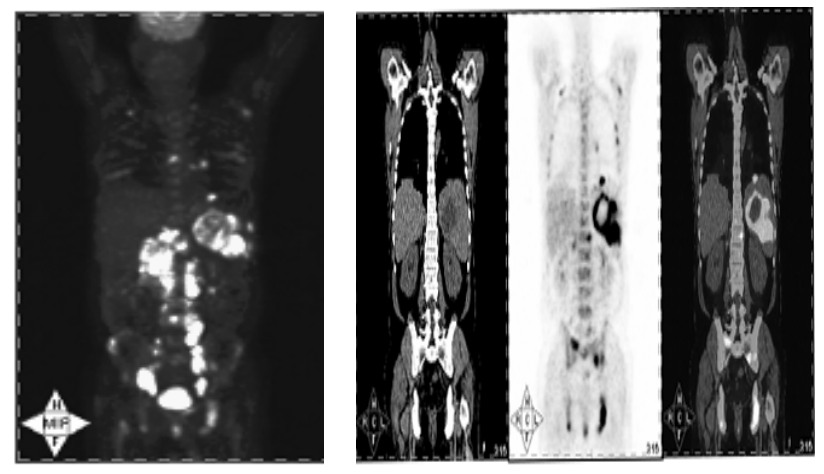

Fig. 1,2. Whole-body PET-CT Scan in a 36 years male diagnosed as Hodgkin's lymphoma (coronal view) shows multiple focal areas of intense FDG uptake in bone marrow, spleen, and abdominal lymph nodes.

help to better define radiation treatment volumes in both early and more advanced disease stages by better defining gross tumour volume. Metabolic activity within a node on PET is not directly reliant on nodal size to determine the presence or absence of malignancy. Nodes that are not enlarged can be shown to contain tumour on FDG PET images, and nodes that are enlarged can be shown to be reactive in nature. For this reason, PET has been shown to be more sensitive and specific than CT for identification of sites of disease (Fig-3). Demonstration of disease in normal-

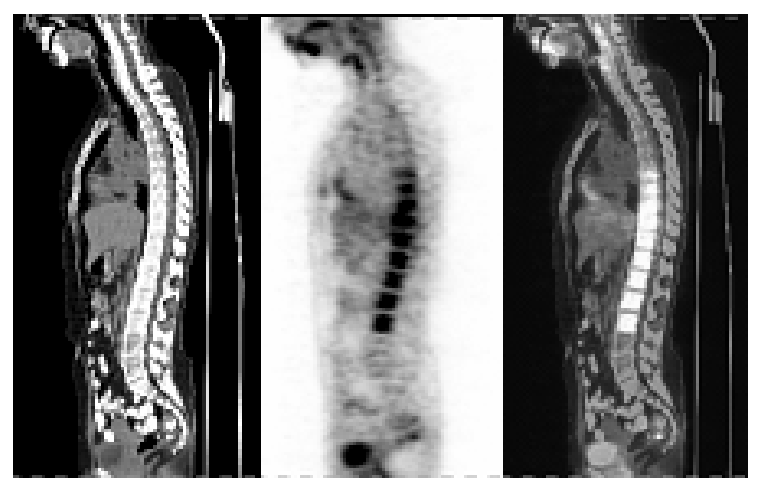

Fig. 3. Whole-body PET-CT scan in a 22-year female Non- Hodgkin's lymphoma Sagital views increased FDG uptake in multiple thoraco-lumber vertebrae suggestive of lymphomatous involvement.

sized lymph nodes could be of particular importance for planning radiation therapy. ${ }^{8-9}$ $\mathrm{PET}$ is also accurate for the identification of specific nodal sites of disease. In a study of 740 lymph node regions in 60 patients evaluated with both FDG PET and CT, discordant interpretations between PET and CT images were almost always resolved in favor of the PET interpretation when confirmation was obtained. ${ }^{10}$ In one study when PET was used to identify the presence or absence of disease in patients with HL both during initial staging and during restaging, it was found to be $86 \%$ sensitive and $96 \%$ specific, compared with $81 \%$ sensitivity and $41 \%$ specificity of CT Scan. ${ }^{8}$ $\mathrm{PET} / \mathrm{CT}$ is superior to CT both for nodal evaluation and detection of extranodal disease. (Table 1). 
Table 1: Value of FDG PET in Malignant lymphoma:

\begin{tabular}{|l|l|l|}
\hline Diagnosis & Role of PET & Comment \\
\hline Initial staging & Limited role & $\begin{array}{l}\text { False positive in chronic inflammatory disease } \\
\text { \& False positive in low grade lymphomas } \\
\text { Changes treatment in 24\% of patients compared } \\
\text { to CT }\end{array}$ \\
\hline $\begin{array}{l}\text { Restaging and } \\
\text { prognostication }\end{array}$ & Good & $\begin{array}{l}\text { Post therapy -ve PET scan 100\% relapse free rate } \\
\text { for 9months. Distinguishes fibrosis and viable } \\
\text { tumour. }\end{array}$ \\
\hline $\begin{array}{l}\text { Early } \\
\text { chemotherapy } \\
\text { response }\end{array}$ & Good & \\
\hline
\end{tabular}

\section{RESTAGING AND PROGNOSIS}

Lymphomatous masses frequently respond dramatically to therapy, with marked reduction in size after effective treatment. Decrease in size of lymphomatous mass in post-treatment CT/MRI compared with pretreatment CT/MRI scan is considered as response to treatment. However, this decrease in size may take a long time or may not occur in cases of fibrosis and necrosis. In many patients, however, there is a residual mass after therapy, and the differentiation between residual viable lymphoma and post treatment necrosis and fibrosis is a challenge with CT or other anatomic imaging modalities. These residual masses are seen in up to $85 \%$ of patients treated for HL and in up to $40 \%$ of those treated for NHL. ${ }^{11-12}$ Recent studies have demonstrated use of PET in treatment evaluation. ${ }^{13}$ FDG PET is able to distinguish between post treatment fibrosis and viable tumour. (Fig.-4). However, PET alone has problem of exact localization. In a retrospective study of 27 patients, all 15 patients with residual biopsy-proven disease and 11 of 12 patients who were disease free were correctly identified with ${ }^{18} \mathrm{~F}$-FDG PET with higher specificity (92\% vs. $17 \%, P<0.01$ ), accuracy ( $96 \%$ vs. $63 \%, P<$ $0.05)$, and positive predictive value ( $94 \%$ vs. $60 \%, P<0.05$ ) compared to CT. ${ }^{13}$ Mikhaeel et al. compared the accuracy of PET with that of CT in the assessment of remission in 49 patients with aggressive NHL ${ }^{14}$ Relapse rates were $100 \%$ for patients with positive PET findings and only $17 \%$ for those with negative PET findings. In a retrospective study of 75 patients with HL or aggressive NHL treated with standard chemotherapy regimens with or without radiation therapy whose disease was restaged with PET and CT, a correlation was found between positive findings on restaging PET and clinical relapse. ${ }^{15}$ Despite the mild physiologic (18) F-FDG uptake in the GIT, F-FDG PET has potential value in monitoring the treatment response in patients with GIT lymphomas, particularly when pretreatment PET results are positive. A positive (18) F-FDG PET scans after the completion of chemotherapy is a strong predictor of relapse. $^{16}$

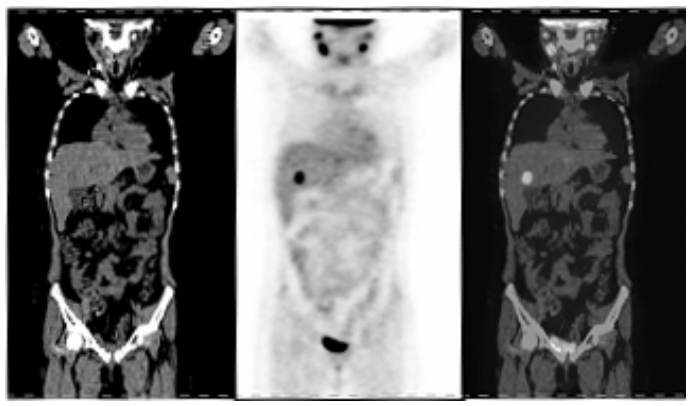

Fig. 4. 11-year female with Hodgkin's Lymploma, underwent chemotherapy and bone marrow transplant. Coronal views of whole body CT, PET and PET-CT show solitary focal area of intense FDG uptake in right lobe of liver suggestive of residual/recurrent disease. 
Cheson et al has recommended revised response criteria in malignant lymphoma considering recent advance techniques like FDG-PET, immuno-histochemistry (IHC) and flow cytometry. This International Harmonization Project was convened to provide updated recommendations defining response in non-Hodgkin's and Hodgkin's lymphoma. ${ }^{17}$

For predictably PET avid histologies (PA) eg. diffuse large B-cell NHL (DLBCL), HL, follicular (FL) and mantle cell lymphoma (MCL), pretreatment $\mathrm{PET}$ is strongly encouraged to define sites of disease. For histologies not predictably PET+ (NPA), PET should only be done, if response is an endpoint. The new definition for complete response (CR) includes: 1) no signs or symptoms of disease; 2) PET negative in a PA lymphoma, or negative CT in NPA lymphoma. 3) Normal bone marrow by morphology, or if indeterminate, negative by IHC, flow and/or molecular genetic studies. Partial response (PR) is defined as 1) $\geq$ $50 \%$ decrease in tumour size, but PET + at prior PA sites, or 2 ) $\geq 50 \%$ decrease in tumour size, but $\mathrm{CT}+$ and PET- if PET- prior to treatment. Bone marrow is irrelevant if positive pre-treatment. Stable disease is neither PR nor progressive disease, PET+ only at prior sites of disease. Progressive/ relapsed disease requires $\geq 50 \%$ increase in disease or new lesions that are PET+ if PA lymphoma. PET does not replace a biopsy before initiating new therapy. ${ }^{17-18}$

Imaging subcommittee of the International Hormonization project has developed consensus recommen-dations based both on published PET literature and on the collective expertise of its members in the use of PET in lymphoma. ${ }^{18}$ The Specific recommendations of this Imaging subcommittee are as follows:

口 After treatment completion, PET should be performed at least 3 weeks and preferably 6 to 8 weeks, after chemotherapy or chemoimmunotherapy, and 8 to 12 weeks after radiation or chemoradiotherapy.
( Visual assessment alone is sufficient for interpreting PET findings as positive or negative when evaluating response after completion of therapy.

( Mediastinal blood-pool activity is recommended as the reference background activity to define PET positivity for a residual mass of $2 \mathrm{~cm}$ in greatest transverse diameter, regardless of its location.

( The criterion for a smaller residual mass or a normal-sized lymph node $(1 \mathrm{x} 1 \mathrm{~cm}$ in diameter) to be considered positive is activity above that of the surrounding background.

The guidelines also discuss recommendations related to the standardization of PET-imaging parameters relevant to response assessment of cancer treatment in general, including patient preparation and image acquisition. . $^{17-18}$

Once therapy is completed, PET offers prognostic information based on the presence or absence of residual FDG-avid disease. In a metaanalysis an FDG PET scan showing metabolically active tumour after treatment was found to be a strong predictor of relapse, with up to $100 \%$ of patients with persistent disease after therapy having recurrent disease within 2 years. ${ }^{19}$

\section{PET FOR EARLY ASSESSMENT OF TREATMENT RESPONSE}

Evaluation of chemotherapy response is an important aspect for the management of these patients, which can be assessed by clinical, imaging and histopathological criteria. Changes in FDG uptake have been reported as early as 13 days after the initiation of therapy, and SUV values at 42 days after treatment have been found to accurately reflect the patient's overall disease status. In a study of 121 patients with NHL, PET was performed before and after $2-3$ 
cycles of chemotherapy and response was correlated to progression-free survival and overall survival (Fig.-5). The median follow-up interval for all patients was 24.4 month. For patients with negative and positive PET findings 5-year progression-free survival was $88 \%$ and $16.2 \%$, respectively. In this study on subgroup analysis for DLBC subtype of NHL results were similar. ${ }^{19}$ In another study ${ }^{18}$ F-FDG performed before and after 1 cycle of chemotherapy in 30 patients with NHL or HL. Scans were scored as positive if the focal uptake was relatively higher than surrounding background activity. 15 patients had residual ${ }^{18}$ F-FDG-avid disease after 1 cycle of therapy. 13/ $15(87 \%)$ patients either experienced relapse or never achieved remission. The remaining 2 patients were in clinical remission for at least 18 month after therapy. Negative PET findings in this study were highly predictive of remission, with $87 \%$ of the patients in complete remission after a median of 19 months of follow-up. ${ }^{20}$

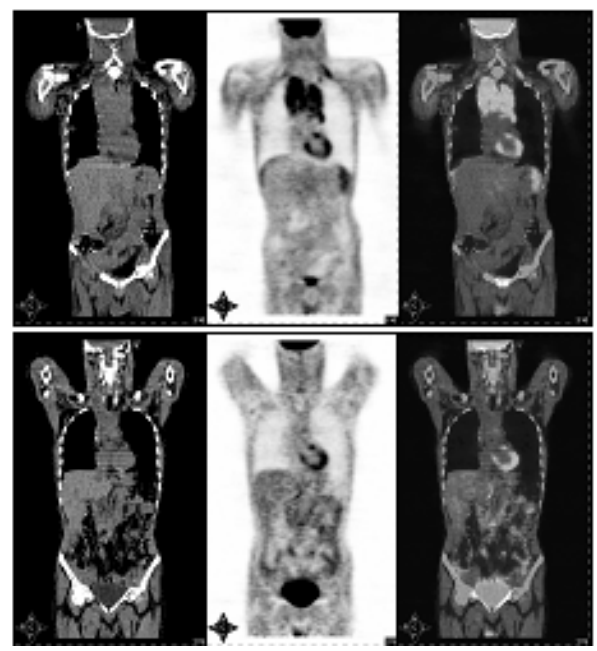

Fig. 5. Whole-body PET-CT scan in a 22 years male with Non- Hodgkin's lymphoma (baseline and post chemotherapy) Coronal views: (upper row) CT, PET and PET-CT show multiple focal areas of intense FDG uptake in mediastinal lymph nodes suggestive of involvement. Coronal views of whole body postchemotherapy (lower row) CT, PET and PET-CT show no FDG uptake in mediastinal lymph nodes suggestive of complete resolution.
PET-CT FOR EVALUATION OF CHEMOTHERAPY RESPONSE IN PATIENTS WITH HIGH-GRADE LYMPHOMAS: AIIMS EXPERIENCE

FDG- PET/CT was carried out to evaluate response to therapy in 52 patients with Hodgkin's and non-Hodgkin's lymphoma (NHL). Fifty-two patients of lymphoma (25 HL, 27 high-grade NHL) with mean age of 43.2 years (age range 14-76 years) (32 male, 20 female) were included in this study. Forty of 52 patients had only nodal disease whereas 12 had extra nodal disease. All patients were fasted for 4 hours prior to the PET-CT scan. PET-CT imaging was performed using a (Biograph, Siemens). PET-CT scanner Scanning was done at approximately 60 minutes after FDG injection of about $10 \mathrm{mCi}$. After image reconstruction, a region of interest (ROI) was carefully drawn around the site of the abnormal FDG uptake in the consequent 4-6 PET-CT scan slices. The slice with a maximal FDG uptake in the ROI was chosen for quantitative measurement of metabolic activity of the tracer (SUV). From these ROIs, the SUV were calculated according to the formula described below.

Mean ROI activity $(\mathrm{MBq} / \mathrm{g})$ / Injected dose $(M B q) / B o d y$ weight $(g)$

Whereas, ' $\mathrm{MBq}$ ' = a mega-Becquerel, and ' $\mathrm{g}$ ' = grams.

Both average and maximum SUV values were calculated for each ROI. All patients underwent a baseline PET-CT scan (prechemotherapy) and follow-up PET-CT scan (postchemotherapy). Follow-up scan was done 2 weeks to 5 months after completion of last cycle of chemotherapy. Both the PET-CT studies were analysed (qualitatively and semiquantitatively) and compared for any change in FDG uptake. Complete response (CR) was seen in 33 (19 HL, $14 \mathrm{NHL})$ patients, partial resolution in $12(6 \mathrm{HL}, 6 \mathrm{NHL})$ and no improvement or deterioration in 7 (all NHL) 
patients. There was good correlation between PET-CT findings with clinical assessment in all patients. All the 19 patients with partial or no improvement underwent more aggressive therapy. Six of these 19 showed CR in follow-up scan (Fig-6). Two patients were found to be false positive. In addition, 9 patients showed parenchymal inflammatory/infective lesion in the both the lungs, which were not seen in the pre chemotherapy, scans. These lesions were attributed to an inflammatory/infective disease process as a result of chemotherapy-induced myelosuppression.

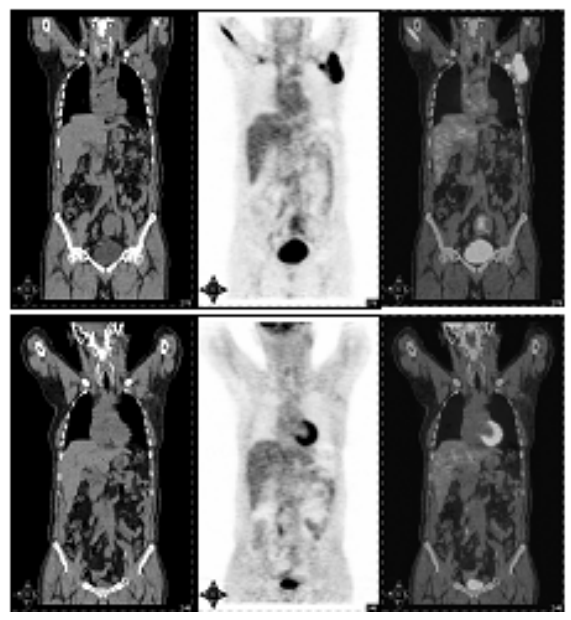

Fig. 6.A 59 year's old male with NHL stage IIA. Pre and post chemotherapy whole-body PET-CT scan. Coronal views (upper row) show multiple focal areas of intense FDG uptake in left axillary lymph nodes suggestive of involvement lower row show no FDG uptake in left axillary lymph nodes suggestive of good response.

\section{OTHER PET TRACERS}

${ }^{18} \mathrm{~F}-3$ '-deoxy-3'-fluorothymidine(FLT) is a marker of cellular proliferation. This is being investigated as an alternative to ${ }^{18} \mathrm{~F}$-FDG PET. Bone marrow uptake of ${ }^{18} \mathrm{~F}$-FLT may reduce its sensitivity; however initial studies have demonstrated promise in imaging NHL. One advantage of ${ }^{18} \mathrm{~F}$-FLT PET is that it does not accumulate in areas of inflammation (which can be common in this particular patient population after radiation or infection) and thus may reduces the false-positive rate. ${ }^{21}$

In conclusion, PET-CT play important role in the assessment of response of treatment in patients with high-grade lymphoma. A negative study is associated with disease-free period not requiring further therapy, even when residual mass is detected on CT. A positive PET-CT scans after the completion of therapy is a strong predictor of residual/recurrent disease. The diagnostic accuracy of PET-CT scans is superior to $\mathrm{CT}$ scans in evaluating the presence of residual disease after the end of treatment. Identification of patients with sub optimal response with PET-CT may substantially influence future treatment strategies in such clinical settings.

\section{REFERENCES:}

2

2. imaging Hodgkin's disease with computed
tomography and positron emission tomography using fluorine-18-fluorodeoxyglucose. Ann Oncol 2001;12:719-22.

7. Vinnicombe SJ, Reznek RH. Computerised tomography in the staging of Hodgkin's disease and nonHodgkin's lymphoma. Eur J Nucl Med Mol Imaging 2003;30(Suppl 1):S42- 55.

Stumpe KD, Urbielli $M$, Steinert HC, Glanzmann $C$, Buck A, von Schulthess GK. Whole-body positron emission tomography using fluorodeoxyglucose for staging of lymphoma: effectiveness and comparison with computed tomography. Eur J Nucl Med 1998;25:721-28. 
Hernandez-Maraver D,Hernandez-Navarro F, GomezLeon $N$, et al. Positron emission tomography/ computed tomography: diagnostic accuracy in lymphoma. Br J Haematol. 2006;135(3):293-302.

10. Moog F, Bangerter $M$, Diederichs $C G$, et al. Lymphoma: role of whole-body 2-deoxy-2-[F-18]fluoroD-glucose (FDG) PET in nodal staging. Radiology 1997;203:795-800.

11. Cremerius U, Fabry U, Neuerburg $J$, et al. Positron emission tomography with ${ }^{18} \mathrm{~F}-\mathrm{FD} G$ to detect residual disease after therapy for malignant lymphoma. Nucl Med Commun. 1998;19:1055-63.

Talbot JN, Haioun C, Rain JD, et al. [18F]-FDG positron emission tomography in clinical management of lymphoma patients. Crit Rev Oncol Hematol 2001;38:193-21.

13. Kumar R, Alavi A. Fluorodeoxyglucose-PET in the management of lymphoma. Radiol Clin North Am. 2004; $42: 1113-22$.

14. Mikhaeel NG, Timothy AR, O'Doherty MJ, Hain $S$, Maisey MN. 18-FDG-PET as a prognostic indicator in the treatment of aggressive non-Hodgkin's lymphoma: comparison with CT. Leuk Lymphoma. 2000; 39:543-53.

15. Zinzani PL, Fanti $S$, Battista $G$, et al. Predictive role of positron emission tomography (PET) in the outcome of lymphoma patients. $\mathrm{Br} J$ Cancer. 2004:91:850-54
16. Kumar R, Xiu Y, Potenta $S$, et al. 18F-FDG PET for evaluation of the treatment response in patients with gastrointestinal tract lymphomas. J Nucl Med. 2004;45:1796-803.

17. Cheson BD, Pfistner B, Juweid ME, et al. The International Harmonization Project on Lymphoma. Revised response criteria for malignant lymphoma Clin Oncol. 2007:10:25(5):579-86.

18. Juweid ME, Stroobants $S$, Hoekstra OS, et al. Imaging Subcommittee of International Harmonization Project in Lymphoma. Use of positron emission tomography for response assessment of lymphoma: consensus of the Imaging Subcommittee of International Harmonization Projectin Lymphoma.J Clin Oncol. 2007;10:25(5):57178.

19. Mikhaeel NG, Hutchings $M$, Fields PA, O'Doherty MJ, Timothy AR. FDG-PET after two to three cycles of chemotherapy predicts progression-free and overall survival in high-grade non-Hodgkin lymphoma. Ann Oncol. 2005;16:1514-23.

20. Kostakoglu L, Coleman M, Leonard JP, Kuji I, Zoe $H$, Goldsmith SJ. PET predicts prognosis after 1 cycle of chemotherapy in aggressive lymphoma and Hodgkin's disease. J Nucl Med. 2002;43:1018-27.

21. Buchmann I, Neumaier B, Schreckenberger $M$, Reske $S$. $\left[{ }^{18} \mathrm{~F}\right]$ 3'-deoxy-3'-fluorothymidine-PET in $\mathrm{NHL}$ patients: whole-body biodistribution and imaging of lymphoma manifestations - a pilot study. Cancer Biother Radiopharm. 2004;19:436-42. 